\title{
Lack of methylation on transgene leads to high level and persistent transgene expression in induced pluripotent stem cells
}

\begin{abstract}
Short-lived therapeutic gene expression in mammalian cells by DNA methylation is one of the major challenges in gene therapy. In this study, we assessed the implication of DNA methylation on the duration of GFP expression in mouse embryonic stem (ES) and mouse induced pluripotent stem (iPS) cells. The cells were transduced with lentivirus (LV) carrying green fluorescent protein (GFP) driven by either human elongation factor (EF1 $\alpha)$ or cytomegalovirus (CMV) promoter. Transduced iPS cells exhibited higher percentage of GFP+ cells with persistent mean fluorescent intensity than transduced ES cells. Analysis on the integrated copy of transgene in the population of the transduced cells demonstrated similar copy number. However, significant increase in GFP intensity following 5-azaC treatment was observed in transduced ES cells only, suggesting the influence of DNA methylation in transgene silencing. Subsequent DNA methylation analysis showed that the promoter and the GFP region of the provirus in iPS cells had negligible methylation profile compared to transduced ES cells. Interestingly, sustained transgene expression was observed upon directed differentiation of transduced iPS cells towards CD34+ CD45+ cells. Hence, this study has shown that favourable transgene activity from lentiviral transduced iPS cells was due to the lack of methylation at the proviral regions.
\end{abstract}

Keyword: Lentivirus; Pluripotent stem cells; DNA methylation; Gene therapy 